\title{
Gait parameters in children with bilateral spastic cerebral palsy: a systematic review of randomized controlled trials
}

Cristina Gómez-Pérez ${ }^{1 *}$, Josep M. Font-Llagunes², Joan Carles Martori Cañas ${ }^{3}$, Joan Vidal Samsó ${ }^{4,5,6}$.

${ }^{1}$ Faculty of Health Sciences and Welfare. University of Vic - Central University of Catalonia (UVic-UCC),

C. Sagrada Família, 7, 08500 Vic, Spain

2 Biomechanical Engineering Lab, Department of Mechanical Engineering and Research Centre for Biomedical Engineering, Universitat Politècnica de Catalunya, Av. Diagonal 647, 08028 Barcelona, Spain

${ }^{3}$ Data Analysis and Modeling Research Group, Department of Economics and Business, Faculty of Business and Communication Studies, University of Vic - Central University of Catalonia (UVic-UCC), C. Sagrada Família, 7, 08500 Vic, Spain

${ }^{4}$ Institut Guttmann, Neurorehabilitation Institute, Camí de Can Ruti, 08916 Badalona, Spain

${ }^{5}$ Universitat Autònoma de Barcelona, Plaça Cívica, 08193 Cerdanyola del Vallès, Spain

${ }^{6}$ Fundació Institut d'Investigació en Ciències de la Salut Germans Trias i Pujol, Carretera Canyet, 08916 Badalona, Spain

WORD COUNT: 4856

*CORRESPONDING AUTHOR: Cristina Gómez-Pérez, Faculty of Health Sciences and Welfare. University of Vic - Central University of Catalonia (UVic-UCC), C. Sagrada Família, 7, 08500 Vic, Spain; E-mail: cristina.gomez1@uvic.cat 


\section{Gait parameters in children with bilateral spastic cerebral palsy: a systematic review of randomized controlled trials}

\section{ABSTRACT}

Aim: To identify the gait parameters used to assess gait disorders in children with bilateral spastic cerebral palsy (BSCP) and evaluate their responsiveness to treatments.

Method: A systematic search within Pubmed, Web of Science and Scopus (English, 2000-2016) for randomized controlled trials of children with BSCP who were assessed by instrumented gait analysis (IGA) was done. Data related to participants and study characteristics, risk of bias and outcome measures were collected. A list of gait parameters responsive to clinical interventions was obtained.

Results: Twenty-one articles met the inclusion criteria. Eighty-nine gait parameters were identified and fifty-six of them showed responsiveness to treatments. Spatiotemporal and kinematic parameters were widely used compared to kinetic and surface electromyography data. The majority of responsive gait parameters were joint angles at the sagittal plane (flexion-extension).

Interpretation: The IGA yields responsive outcome measures for the gait assessment of children with BSCP. Spatiotemporal and kinematic parameters are the gait parameters used most frequently. Further research is needed to establish the relevant gait parameters for each clinical problem.

SHORTENED FORM OF THE TITLE: Gait Analysis in Cerebral Palsy Children.

\section{WHAT THIS PAPER ADDS}

- Fifty-six responsive gait parameters for children with bilateral spastic cerebral palsy were identified.

- Most responsive gait parameters belong to joint angles time-series at sagittal plane.

- Spatiotemporal and kinematic parameters are widely used compared to kinetic and SEMG parameters.

- Further efforts improving the clinical management of kinetic and SEMG data are required.

- Further research to establish the relevant gait parameters for each clinical problem is needed. 


\section{INTRODUCTION}

Cerebral palsy (CP) is the most common cause of chronic childhood motor disability ${ }^{1}$ with a prevalence of above 2.0 per 1000 live births $^{2}$. CP describes a group of permanent disorders affecting movement and posture and causing activity limitation that are attributed to non-progressive lesions in the developing fetal or infant brain ${ }^{3}$. The motor disorders of CP often occur together with disturbances of sensation, cognition, communication and behaviour, with epilepsy and with secondary musculoskeletal problems ${ }^{3}$.

Spasticity is often the dominant motor disorder ${ }^{4}$, along with loss of selective motor control and impaired balance ${ }^{5}$, and it can be classified according to different topographical patterns such as quadriplegia, diplegia and hemiplegia, with additional terms such as monoplegia and triplegia, or unilateral and bilateral ${ }^{6}$. Secondary musculoskeletal problems like muscle contractures, bony deformities and joint instability appear as a consequence of growth and development of the musculoskeletal system ${ }^{5}$. Their interaction, occurring at multiple levels, affects the quality and efficiency of gait and other aspects of motor function, contributing to activity limitation and participation restriction ${ }^{5,7}$.

The Gross Motor Function Classification System (GMFCS) has been universally adopted to describe the movement ability of children with $\mathrm{CP}^{8}$. Its expanded and revised version uses five ordinal levels across five age bands, with emphasis on the typical performance in different settings ${ }^{9}$. The general headings for each level are: walks without limitations (I); walks with limitations (II); walks using a hand-held mobility device (III); self-mobility with limitations, may walk with physical assistance or use powered mobility (IV); and transported in a manual wheelchair $(\mathrm{V})^{10}$. Children with unilateral CP almost always develop independent locomotion; in the case of bilateral CP, some children walk independently, some walk with aids and others can never achieve this function ${ }^{11}$. Considerable research effort has been directed towards improving or maintaining walking ability of children with CP through different clinical interventions such as surgery, pharmacology, orthotics or physical therapy ${ }^{5,12}$. Using reliable, valid and responsive outcome measures to evaluate the success of these interventions is crucial ${ }^{12}$.

The Instrumented Gait Analysis (IGA) allows a precise quantification of gait characteristics, through objective data that cannot be appreciated visually or measured on a static physical examination ${ }^{13}$. The IGA provides detailed information on four main types of data recorded simultaneously: spatiotemporal, kinematic, kinetic and surface electromyography (sEMG) parameters ${ }^{14,15}$. The IGA is often used in the assessment of ambulatory children with cerebral palsy ${ }^{16}$, for multiple purposes including the identification and understanding of gait deviations, the refinement of clinical decisionmaking and the evaluation and understanding of the effects of treatments on gait deviations $\mathrm{s}^{5,13,14}$. However, the reliability, validity and clinical utility of the IGA have not been well established ${ }^{5}$ and its clinical use remains variable and controversial ${ }^{15,17}$.

One of the handicaps of the IGA is the large amount of data collected and analyzed, that makes it an instrument complicated to use and difficult to interpret ${ }^{18}$. A methodology for properly interpreting data from the IGA has not been defined clearly ${ }^{15}$ so clinicians and researchers have the challenge to extract the clinically relevant information from this large amount of data ${ }^{19}$. Kinematic and kinetic data analysis can be performed in two different ways: scalar gait parameters analysis and full gait waveforms analysis ${ }^{19}$. Scalar gait parameters analysis (which refers to specific vector components in 
specific time instants of the gait cycle) is the most frequently used in intervention studies, but there is no consensus on which parameters should be evaluated ${ }^{19}$. Different methods have been used to select relevant gait parameters, from conventional manual procedures based on subjective available clinical expert knowledge to novel automated procedures based on objective mathematical techniques ${ }^{20}$.

Two requirements for a clinically relevant gait parameter are: 1) its capability to distinguish between physiological and pathological gait (clinical problem characterization), and 2) its capability to separate between two therapy stages within the same patient group (responsiveness to treatments) ${ }^{20}$. The responsiveness (ability to assess significant changes) of gait parameters to interventions should be established with caution due to the risk of type I (false positive: the mistake of inferring an experimental effect when none exists in reality) ${ }^{21}$ and type II (false negative: the mistake of missing real effects) ${ }^{22}$ errors. Evidence to support the responsiveness of gait parameters is needed ${ }^{12}$.

The objective of this systematic review is to critically evaluate and summarize the current evidence base related to the clinical use of the IGA for the assessment of gait disorders in children with bilateral spastic cerebral palsy (BSCP). We aim to identify the gait parameters most widely used and evaluate their responsiveness to treatments for children with BSCP.

\section{METHOD}

\subsection{Search strategy}

In order to identify the key articles on this topic, a systematic search was undertaken within the following online databases: PubMed, Web of Science and Scopus. Constraints were applied for year of publication (2000-2016), language (English) and document type (clinical trial). Search through PubMed was also limited for species (humans) and text availability (abstract). The user query used was: (cerebral palsy OR spastic diplegia) AND (child OR adolescent) AND (gait OR walking OR ambulation OR locomotion) AND (spatiotemporal parameters OR kinematics OR kinetics OR electromyography OR three-dimensional gait analysis OR 3D gait analysis OR instrumented gait analysis OR quantitative gait analysis OR computerized gait analysis).

\subsection{Eligibility criteria}

Articles were included if they satisfied the following criteria: 1) Randomized controlled trials (RCT) with statistical analysis of the results; 2) Percentage of subjects with diagnosis of BSCP > 60\%; 3) Mean age of the sample between 6 and 18 years old; 4) IGA for obtaining outcome measures, including spatiotemporal, kinematic, kinetic and/or sEMG parameters.

\subsection{Risk of bias}

To check the validity of the RCT selected, the Cochrane risk of bias too ${ }^{23}$ was used. This tool allows analyzing the adequacy of different features related to the risk of bias: random sequence generation, allocation sequence concealment, blinding, incomplete outcome data and selective outcome reporting. Included studies were asked to be RCT so we firstly analyzed the way randomization was carried out (participants' selection bias). On the other hand, this review aimed to evaluate the responsiveness of gait parameters to treatments so we secondly analyzed the risk of type I and type II errors due to the gait parameters' selection bias. The analysis of the risk of bias involves answering "low risk of bias", "high risk of bias" or "unclear" (lack of information or uncertainty over the potential for bias) ${ }^{23}$. 


\subsection{Data collection}

A data extraction sheet was developed, pilot-tested on the 14 first included studies and refined accordingly. Firstly, information related to participants and study characteristics was extracted in order to establish the comparability of the included studies: eligibility criteria, participants, study design, intervention and assessment. Secondly, gait parameters were classified, according to their nature, in spatiotemporal, kinematic, kinetic, sEMG and summary indexes, and their significant results (intra- or intergroup statistical analysis) were collected in order to determine their responsiveness to interventions. Both text and tables data were considered. Only outcomes which were statistically analyzed, and significant results obtained from randomized interventions were included. Significant results obtained from a combination of experimental and control group data, and kinematic parameters calculated from video observation were excluded. Finally, results were summarized in tables.

\subsection{Additional analysis}

From the data collection, an additional analysis was performed to study the responsiveness of gait parameters to different treatments. In this analysis, interventions were grouped into different types and the gait parameters that showed significant results for each type of intervention were determined.

\section{RESULTS}

\subsection{Study selection}

The search of Pubmed, Web of Science and Scopus databases provided a total of 334 citations, taking into account the above-mentioned user query and the search constraints. The last search was run on August $10^{\text {th }}, 2017$. After adjusting for duplicates, 199 remained. After reviewing the title and the abstract, 150 studies were discarded because they clearly did not meet the inclusion criteria. The full text of the remaining 49 studies was examined in more detail. Finally, 21 studies met the inclusion criteria and were included in the literature review ${ }^{24-44}$. In one article ${ }^{34}$, only one of the studies (phase I) was included. All the studies finally selected for the review were RCT published in English in the period 2000-2016. See the flow diagram in Figure 1.

\subsection{Trial's eligibility criteria}

The inclusion criteria of participants in the different studies include information related to diagnosis (21 studies), age range (16), gross motor function (20), range of motion (nine), severity of motor disorders (spasticity and/or muscle weakness; six), secondary musculoskeletal problems (contractures and deformities; 14), medical history (surgery, drugs and/or rehabilitation; 19), sensory impairments (visual, auditory or perceptual; seven), degree of comprehension (13), anthropometric measures (height and/or weight; three) and treatment contraindications (four).

\subsection{Participants}

The included studies involved a total of 528 children with spastic cerebral palsy. The majority of the participants had a diagnosis of BSCP ( $n=488,92 \%)$, at least $419(79 \%)$ spastic diplegic, and the mean age of the children was 8 years 7 months. The ability to walk of the participants was mainly defined through the GMFCS. Seven studies only included participants with independent walking (GMFCS levels I and/or II), 10 studies included participants able to walk with or without walking aids (GMFCS levels I, 
II and/or III) and four studies additionally included participants able to walk with external support (GMFCS levels I, II, III and/or IV) (see Table 1).

Other characteristics were detailed only in some studies, for example, gender (15 studies), anthropometric measures like weight, height or body mass index (10), the gait pattern (five), the use of walking aids or orthosis (three) and the history of surgery, physical therapy or botulinum toxin $A$ (BTX-A) injections (three).

\subsection{Study design}

Eighteen studies used a parallel group design: different interventions were applied to at least two different groups (experimental and control groups). The other three studies used a cross-over design: there were two different interventions ( $A$ and $B$ ) and all the children received both interventions in a randomized order. Two studies defined a healthy control group but only data from the cerebral palsy groups was taken into account in the review (see Table 2).

\subsection{Intervention}

A big variety of interventions were studied in the included studies: surgical procedure (single event multilevel surgery (SEMLS), distal rectus femoris transfer and/or selective dorsal rhizotomy; four studies), BTX-A (four), casting (four), orthopedic device (ankle-foot orthosis, strapping system and/or postural insole; three), individually defined physical therapy (one), strength training program (whole body vibration training, resistance and/or active exercises or neuromuscular electrical stimulation; five), balance training program (one), gait training program (gait trainer, treadmill training or partial body-weight-supported treadmill training; three), hippotherapy (one) and transcranial direct current stimulation (one) (see Table 2).

\subsection{Instrumented gait analysis}

All included studies assessed participants at least twice. Eight studies made pre- and post-intervention assessments, seven studies made pre-intervention and follow-up assessments and four studies made pre-, post-intervention and follow up assessments. Three studies made assessments in different conditions: with and without the intervention device (see Table 2).

When performing the IGA, different measurement tools were used synchronously (integrated solutions) or independently: three dimensional gait analysis (3DGA) system, force plate, sEMG and video recording. 3DGA was used in 19 studies to obtain kinematic and/or spatiotemporal parameters. The number of infrared cameras went from five to 16 (six being the most common) and the recording frequency from 100 to $120 \mathrm{~Hz}$. The markers were reflective with a diameter between 9 and $25 \mathrm{~mm}$. Eight studies used force plates to obtain kinetic data and the number of platforms went from one to three (two being the most common). Five studies used SEMG to obtain muscle activation data, and information about channels supported, sample frequency, amplifier, transmitter, filters (high-pass and low-pass) and electrodes (type, area and inter-electrodes distance) was reported. Eight studies used a video system as a complement to the other measurement tools (see Table 2).

In all the studies, participants were asked to walk on a walkway. In 12 studies, the length of the walkway was specified, with a mean value of $8 \mathrm{~m}$. In 11 studies, the minimum number of walking trials (collected and/or selected) was reported, ranging from two to six trials. Two studies reported the maximum number of walking trials (eight and 10 respectively). Fifteen studies reported the walking 
speed that was indicated to participants. In all cases, self-selected walking speed was chosen. Some studies also described whether participants walked barefoot (eight studies) or with usual footwear (two), with orthosis or insoles (four) and/or with walking aids (five). Ten studies used data from children with typical development as normative reference.

All the studies used the IGA for obtaining outcome measures (it was one of the inclusion criteria of the review). Additionally, the IGA was used to define the gait pattern of the participants (two studies), the rehabilitation devices setup (two) and the BTX-A target muscles (two).

\subsection{Risk of bias}

The risk of bias assessment was focused on the participants' selection and the gait parameters' selection. When assessing the participants' selection bias, the random sequence generation and the allocation concealment were studied. Different techniques were reported in the included studies. Regarding the random sequence generation, the following criteria was applied when analyzing the risk of bias: 1) computer random number generation, minimization and block randomization with block size masked were considered as "low risk of bias"; 2) alternation was considered as "high risk of bias"; and 3) envelopes and block randomization without specifying the sequence generation technique were considered as "unclear risk of bias". In relation to the allocation concealment: 1) sequentially numbered, opaque, sealed envelopes and central randomization (performed by a person independent to the study) were considered as "low risk of bias"; 2) alternation was considered as "high risk of bias"; and 3) envelopes with one or two of the requirements (sequentially numbered, opaque and sealed), computer randomization without specifying the allocation method and random allocation schedule without specifying that it was not open were considered as "unclear risk of bias". Three studies showed a low risk of bias in both features and seven studies showed a low risk of bias in one of them (with the other one classified as unclear). In 10 studies, the whole randomization process was classified as unclear and one study showed a high risk of participants' selection bias (see Table 1).

The assessment of the gait parameters' selection bias was based on the ideal hypothesis testing defined in Pataky et al. ${ }^{21}$ and the following criteria were applied: 1 ) directed hypotheses (claim response in specific gait parameters) followed by analyses of the same specific gait parameters and non-directed hypotheses (broadly claim kinematic, kinetic or sEMG response) followed by full gait waveforms analyses were considered as "low risk of bias"; and 2) specific gait parameters analyses following non-directed hypotheses (broadly claim spatiotemporal, kinematic, kinetic or gait response) and directed hypotheses followed by analyses of more specific gait parameters than those defined in the hypotheses were considered as "high risk of bias". We considered as hypothesis the last paragraph of the introduction section of the included studies, independently of the terminology used (hypothesis, aim, objective, goal or purpose). Sixteen studies showed high risk of gait parameters' selection bias, and five studies showed low risk.

No subgroup analyses of the results were done considering the risk of bias results because it is not possible to know if the bias really existed and any judgment could be unfair.

\subsection{Outcomes}


This section summarizes the gait parameters used as outcome measures in the included studies. The reported parameters were classified in spatiotemporal, kinematic ("joint angles" referring to ankle, knee and hip, and "segment angles" referring to foot and pelvis), kinetic, sEMG and summary indexes.

Only three included studies provided detailed parameters definitions ${ }^{26,28,30}$. Gait parameters with different terminology were grouped together if they had a similar meaning (e.g. "minimum knee flexion in stance" ${ }^{\prime 30}$ and "maximum knee extension in stance" ${ }^{43}$ ) and a common terminology was provided in order to homogenize the definition criteria. Sometimes, it was difficult to establish the correct definition for each gait parameter. For example, it is not clear if the gait parameter "ankle angle at initial swing" ${ }^{\prime 31}$ refers to a specific time instant of the gait cycle (toe off) or to the mean value during a gait subphase (initial swing). Some spatiotemporal parameters were defined according to Grecco et al. $^{28}$. The nomenclature of kinematic and kinetic parameters was divided in three different items related to their definition: value, time-series and gait phase (e.g. the minimum value of the hip flexionextension angle at stance phase was named MIN_HipFlexExt_St), based on Wolf et al. ${ }^{20}$, and a short definition was given for each item. The definition of the summary indexes was also provided (see Table 3). sEMG data was cataloged by muscles, independently of the statistical parameter used in each study.

For each parameter, it was determined whether statistically significant differences were observed, either in the intra- or intergroup analysis, considering a $p$-value $<0.05$.

\subsubsection{Spatiotemporal parameters}

Eighteen studies analyzed spatiotemporal data. Seven different parameters were reported: gait speed (17 studies), cadence -also expressed as cycle time- (15), stride length -also expressed as step length(17), step width (two), time of toe off -also expressed as stance phase or swing phase- (six), single support (one) and double support (one). Gait speed was calculated in $\mathrm{m} / \mathrm{s}, \mathrm{cm} / \mathrm{s}$ or m/min (15 studies) and it was also normalized to account for leg length (one). Cadence was calculated in steps/min or cycles/min (10 studies) and, when expressed as cycle time, in s or ms (four). Stride length was calculated in $\mathrm{m}$ or $\mathrm{cm}$ (13 studies) and percentage of height (one). Time of toe off, single support and double support were calculated in percentage of cycle. Statistically significant changes $(p<0.05)$ within groups (intra-group analysis) and/or between groups (inter-group analysis) were observed for five spatiotemporal parameters: gait speed (11 studies), cadence (seven), stride length (nine), time of toe off (one) and single support (one) (see Table 4).

\subsubsection{Kinematic parameters}

Fifteen studies analyzed kinematics of the lower limb, including segment angles: foot (four studies) and pelvis (five); and joint angles: ankle (12), knee (13) and hip (10); in the three planes: sagittal (15), frontal (four) and transverse (four). Four studies analyzed kinematics at the five levels (foot, ankle, knee, hip and pelvis) and in the three planes (sagittal, frontal and transverse). There were 64 different kinematic parameters explicitly reported: foot (four parameters), ankle (14), knee (18), hip (19) and pelvis (nine); sagittal plane (44), frontal plane (eight) and transverse plane (12). Significant changes were found in 38 kinematic parameters: foot (three parameters), ankle (10), knee (13), hip (eight) and pelvis (four); sagittal plane (30), frontal plane (three) and transverse plane (five) (see Tables 4 and 5).

\subsubsection{Kinetic parameters}


Five studies analyzed kinetics, including ankle, knee and hip moment (five studies) and power (three) in the sagittal plane. Joint moment was calculated in $\mathrm{N} \cdot \mathrm{m} / \mathrm{kg}$ (normalized to body mass) (four studies) and joint power in $\mathrm{W}$ (one) or $\mathrm{W} / \mathrm{kg}$ (two). Eight different parameters were reported: PlantDorsFlexMo_IC (one study), MAX_PlantDorsFlexMo_LR (two), MAX_PlantDorsFlexMo_POff (five), MIN_AnkleGenAbsPo_LR (one), MAX_AnkleGenAbsPo_POff (three), MIN_KneeGenAbsPo_LR (one), MIN_HipGenAbsPo_St (one) and MAX_HipGenAbsPo_St (one). Significant changes were observed in four kinetic parameters: MAX_PlantDorsFlexMo_LR (one study), MAX_PlantDorsFlexMo_POff (two), MIN_AnkleGenAbsPo_LR (one) and MAX_HipGenAbsPo_St (one) (see Table 4).

\subsubsection{SEMG parameters}

Four studies analyzed SEMG data. Each one used different parameters related to sEMG: root mean square difference, mean asymmetry score (in $\mathrm{mV}$ ), dynamic EMG score (in percentage of number of patients in which muscle is active during gait cycle) and maximal linear envelope of EMG (dynamic rectified EMG recordings in $\mathrm{mV}$ ). Eight muscle groups were studied: gastrocnemius (three studies), soleus (one), tibialis anterior (two), rectus femoris (two), vastus lateralis (one), lateral hamstrings (one), medial hamstrings (two) and adductor (one). Significant changes within or between groups were found in all the muscles (at least in one study) except in vastus lateralis (see Table 4).

\subsubsection{Summary indexes}

Five studies analyzed one of these two summary indexes: the Gillette Gait Index (GGI) (three studies), and the Gait Profile Score (GPS) (two). Significant changes were found in both indexes (see Table 4).

\subsection{Gait parameters responsiveness to different treatments}

Interventions were grouped in eight different types: surgery, BTX-A + casting, orthopedic devices, strength training, balance training, gait training, individualized therapy and hippotherapy. Surgery produced significant changes in kinematic parameters, mainly at knee (nine parameters), and one summary index (GGI). BTX-A and/or casting showed significant differences in spatiotemporal, kinematic (foot, ankle, knee, hip and pelvis), kinetic (ankle and hip) and SEMG parameters. Orthopedic devices showed significant results in spatiotemporal, kinematic (ankle, knee and hip) and ankle kinetic parameters. Strength training significantly changed spatiotemporal and kinematic (ankle and knee) parameters. Balance training produced significant results in spatiotemporal parameters. Gait training showed significant results in spatiotemporal and kinematic parameters, mainly at hip (five parameters), and one summary index (GPS). Individualized therapy significantly changed spatiotemporal and kinematic (knee and pelvis) parameters. Hippotherapy showed significant changes in SEMG data (adductor muscle activity) (see Table 6).

\section{DISCUSSION}

This work presents a literature review of 21 RCT, published in English between the years 2000 and 2016, that used the IGA to obtain spatiotemporal, kinematic, kinetic and SEMG outcome measures. We identified the gait parameters used to evaluate gait disorders in children with BSCP and analyzed their responsiveness to clinical interventions. 
A total of 89 gait parameters were statistically analyzed in the included studies. Spatiotemporal parameters were the most frequently used (18 included studies) followed by kinematic (15), kinetic (five), summary indexes (five) and SEMG data (four). If the parameters are analyzed individually, gait speed, stride length and cadence were the most frequently used (in 17, 17 and 15 studies, respectively) while the rest of parameters were used only in one study ( $47 \%$ of the gait parameters), two studies (31\%) or between three and six studies (15\%). It should be studied why kinetic and SEMG data are not usually used in intervention studies although they are considered necessary to clarify the gait patterns commonly seen in individuals with $\mathrm{CP}$ and plan an appropriate intervention ${ }^{13}$. Further research is needed to establish the relevance of kinetic and SEMG parameters as outcome measures.

Fifty-six gait parameters showed significant results. Kinematic were the type with more responsive parameters (38) followed by sEMG (seven), spatiotemporal (five), kinetic (four) and summary indexes (two). $81 \%$ of responsive kinematic parameters were joint angles (ankle, knee or hip) and $79 \%$ were from sagittal plane. This makes sense since the widest movements involved in gait are ankle plantarflexion/dorsiflexion, and knee and hip flexion/extension. Most of the gait pattern classifications are based on sagittal plane kinematics ${ }^{14}$ and many gait deviations observed and treated in children with CP occur in the sagittal plane $e^{45}$. However, deviations in the transverse and frontal planes are also considered important in clinical decision-making and intervention planning, and analyses in these planes could improve content validity of gait classifications ${ }^{46}$.

The selection of an appropriate outcome measure depends on many factors including the type of intervention ${ }^{47}$. Responsiveness is intervention-specific so we analyzed the gait parameters that showed significant results for each type of intervention. Gait speed, cadence and stride length showed to be responsive to the majority of interventions or, analyzed from another point of view, the majority of interventions had an effect on them. On the other hand, from the number of gait parameters with significant results, we observed that some interventions had their main effect at a certain level: BTX-A + casting and orthopedic devices on the ankle, surgery on the knee and gait programs on the hip. The studies included in this review were not selected for the analysis of the relationship between gait parameters and interventions. Neither a rigorous scientific methodology was followed to statistically analyze this relationship. Therefore, these results should be considered only as additional observations that could inspire new hypotheses and future research studies on this field.

There is no consensus on the relevant gait parameters for each clinical problem ${ }^{19}$. Only three included studies specified the parameters selection criteria ${ }^{35,39,43}$ (based on the expected changes or a study of the literature), so the selection probably could have been done subjectively in all cases. From the fifteen studies that used kinematic and/or kinetic data, thirteen analyzed specific gait parameters and two analyzed the full time-series through the Gait Variable Score (also called Movement Assessment Profile $)^{26,28}$. There are two main risks when using scalar gait parameters: 1) the rationale behind the selection of the gait parameters is often unclear. Reducing the large amount of data subjectively may introduce post hoc regional focus bias (type I or type II error resulting from expanding or reducing the scope of the clinical hypothesis after seeing the data) and potential clinically relevant parameters could be omitted ${ }^{19,21,48} ; 2$ ) there exist covariance among vector components of multidimensional kinematic and kinetic data. Conducting scalar statistical testing on multiple dependent gait parameters may 
introduce inter-component covariation bias (type I or type II error resulting from the failure to consider the covariance among vector components), especially in small sample sizes ${ }^{19,48,49}$.

Some solutions have been proposed to avoid these risks. First of all, a clear hypothesis should be stated a priori and an adequate statistical approach should be selected in accordance to this hypothesis ${ }^{19}$. In case of non-directed hypotheses ${ }^{48}$, statistical methods such as the Bonferroni correction are often applied to deal with the risk of detecting a false positive when testing a large number of dependent gait parameters, but some of them can increase the probability of obtaining a false negative result ${ }^{19,22}$. The statistical parametric mapping (SPM), which belongs to full gait waveforms analysis, is a promising statistical alternative to scalar gait parameters analysis with regard to the interpretation of multidimensional biomechanical data ${ }^{19,21,48}$. SPM is able to perform hypothesis testing on kinematic and kinetic data in a continuous manner, avoiding the need for subjective a priori data reduction, and it also takes into account the dependency between different time instances of the gait cycle ${ }^{19}$. So, SPM overcomes both bias sources ${ }^{48}$. In case of directed hypotheses ${ }^{48}$, performing a scalar gait parameters analysis overcomes the risk of bias ${ }^{21,48}$.

There is another handicap related to scalar gait parameters: they are usually defined on the basis of normal kinematic and kinetic waveforms and they can be difficult to extract from pathological gait waveform ${ }^{50}$. Furthermore, the definitions of scalar gait parameters are often unclear, making it difficult for researchers to reproduce or confirm results ${ }^{19}$. SPM could be a solution since it avoids the need to define gait parameters. Otherwise, a clear definition of the scalar gait parameters (like the one proposed in this review) could help clinicians to understand, interpret, reproduce and compare results.

The IGA is expensive, complex and time-consuming to learn and to use in real practice ${ }^{5}$. Consequently, it is not always accessible for clinicians ${ }^{51,52}$. The quotidian application of expensive healthcare technologies cannot be justified until the evidence unequivocally demonstrates its utility ${ }^{5}$. Conclusions about the usefulness of the IGA can only come from multiple high quality scientific studies free from bias $^{16}$. However, these studies are scarce ${ }^{15,17}$. Our review provides evidence from RCT supporting the responsiveness of the gait parameters to interventions. Our results may also guide clinicians and researchers to select the most relevant gait parameters according to the clinical hypothesis and the treatment selection. The IGA is one of the many inputs into the clinical decision-making process ${ }^{16}$ and we recommend using it together with usual clinical assessment. In the identification of walking problems, differences are detected when using the IGA or the clinical assessment ${ }^{45}$; the IGA is not a substitute for the clinical assessment but should be used to provide evidence and enhance clinical decision-making ${ }^{13}$. The use of a diagnostic and assessment protocol, based on different sources of information and including the IGA, is crucial to achieve an evidence-based practice to optimize the gait pattern and the gait function of children with cerebral palsy.

Some limitations should be considered when interpreting the findings/results of this review: 1) the scope of this systematic review was limited to English-language RCT, which might have underrepresented the set of gait parameters used worldwide, 2) only one reviewer was involved in the study selection and data collection processes, which might have increased the risk of misinterpretation, and 3) there was a big heterogeneity with regards to the selection and definition of the gait parameters, which made difficult the analysis and comparison of results. 
In conclusion, the IGA yields responsive outcome measures for the gait assessment of children with BSCP. Spatiotemporal and kinematic parameters are widely used in comparison to kinetic and SEMG data. Further research is needed to determine the role of kinetic and SEMG parameters in the gait analysis and to establish the relevant gait parameters for each clinical problem.

ACKNOWLEDGEMENTS: The authors would like to thank Olga Araujo Gutiérrez for her assistance as librarian and Amy Croft for her help in copyediting this review.

CONFLICT OF INTEREST: The authors have stated that they had no interests which might be perceived as posing a conflict or bias.

FUNDING: This study received the support of a predoctoral grant in physiotherapy from the University of Vic - Central University of Catalonia. No benefits in any form have been received or will be received from a commercial party related directly or indirectly to the subject of this article. 


\section{REFERENCES}

1. Pakula AT, Van Naarden Braun K, Yeargin-Allsopp M. Cerebral palsy: classification and epidemiology. Phys Med Rehabil Clin N Am 2009; 20: 425-52.

2. Odding E, Roebroeck ME, Stam HJ. The epidemiology of cerebral palsy: incidence, impairments and risk factors. Disabil Rehabil 2006; 28: 183-91.

3. Rosenbaum $\mathrm{P}$, Paneth $\mathrm{N}$, Leviton $\mathrm{A}$, et al. A report: the definition and classification of cerebral palsy April 2006. Dev Med Child Neurol Suppl 2007; 109: 8-14.

4. Rethlefsen SA, Ryan DD, Kay RM. Classification systems in cerebral palsy. Orthop Clin North Am 2010; 41: 457-67.

5. Narayanan UG. The role of gait analysis in the orthopaedic management of ambulatory cerebral palsy. Curr Opin Pediatr 2007; 19: 38-43.

6. Reid SM, Carlin JB, Reddihough DS. Classification of topographical pattern of spasticity in cerebral palsy: a registry perspective. Res Dev Disabil 2011; 32: 2909-15.

7. McGinley JL, Dobson F, Ganeshalingam R, Shore BJ, Rutz E, Graham HK. Single-event multilevel surgery for children with cerebral palsy: a systematic review. Dev Med Child Neurol 2012; 54: 11728.

8. Reid SM, Carlin JB, Reddihough DS. Using the Gross Motor Function Classification System to describe patterns of motor severity in cerebral palsy. Dev Med Child Neurol 2011; 53: 1007-12.

9. Palisano RJ, Rosenbaum $\mathrm{P}$, Bartlett $\mathrm{D}$, Livingston $\mathrm{MH}$. Content validity of the expanded and revised Gross Motor Function Classification System. Dev Med Child Neurol 2008; 50: 744-50.

10. Palisano R, Rosenbaum P, Walter S, Russell D, Wood E, Galuppi B. Development and reliability of a system to classify gross motor function in children with cerebral palsy. Dev Med Child Neurol 1997; 39: 214-23.

11. Akbaş AN. Assessments and outcome measures of cerebral palsy. In: Gunel MK, editor. Cerebral Palsy: Current Steps. Croatia: InTech; 2016. 23-48.

12. Zanudin A, Mercer TH, Jagadamma KC, van der Linden ML. Psychometric properties of measures of gait quality and walking performance in young people with Cerebral Palsy: A systematic review. Gait Posture 2017; 58: 30-40.

13. Chang FM, Rhodes JT, Flynn KM, Carollo JJ. The role of gait analysis in treating gait abnormalities in cerebral palsy. Orthop Clin North Am 2010; 41: 489-506.

14. Armand S, Decoulon G, Bonnefoy-Mazure A. Gait analysis in children with cerebral palsy. EFORT Open Rev 2016; 1: 448-60.

15. Benedetti MG, Beghi E, De Tanti A, et al. SIAMOC position paper on gait analysis in clinical practice: General requirements, methods and appropriateness. Results of an Italian consensus conference. Gait Posture 2017; 58: 252-60.

16. Theologis T, Wright J. Is 3-D gait analysis essential? By Professor James Wright: Introduction by Mr. Tim Theologis. Gait Posture 2015; 42: 227-9.

17. Wren TAL, Gorton GE, Ounpuu S, Tucker CA. Efficacy of clinical gait analysis: A systematic review. Gait Posture 2011; 34: 149-53.

18. Cimolin V, Galli M. Summary measures for clinical gait analysis: a literature review. Gait Posture 2014; 39: 1005-10. 
19. Nieuwenhuys A, Papageorgiou E, Pataky T, De Laet T, Molenaers G, Desloovere K. Literature Review and Comparison of Two Statistical Methods to Evaluate the Effect of Botulinum Toxin Treatment on Gait in Children with Cerebral Palsy. PloS One 2016; 11: e0152697.

20. Wolf S, Loose T, Schablowski M, et al. Automated feature assessment in instrumented gait analysis. Gait Posture 2006; 23: 331-8.

21. Pataky TC, Vanrenterghem J, Robinson MA. The probability of false positives in zero-dimensional analyses of one-dimensional kinematic, force and EMG trajectories. J Biomech 2016; 49: 1468-76.

22. McLaughlin MJ, Sainani KL. Bonferroni, holm, and hochberg corrections: Fun names, serious changes to P values. PM R 2014; 6: 544-6.

23. Higgins JPT, Altman DG, Sterne JAC (editors). Chapter 8: Assessing risk of bias in included studies. In: Higgins JPT, Green S (editors) Cochrane Handbook for Systematic Reviews of Interventions Version 510 (updated March 2011) [Internet]. The Cochrane Collaboration; 2011. Available from: www.handbook.cochrane.org

24. Neto HP, Grecco LAC, Duarte NAC, et al. Immediate Effect of Postural Insoles on Gait Performance of Children with Cerebral Palsy: Preliminary Randomized Controlled Double-blind Clinical Trial. J Phys Ther Sci 2014; 26: 1003-7.

25. Abd El-Kafy EM, El-Basatiny HMYM. Effect of postural balance training on gait parameters in children with cerebral palsy. Am J Phys Med Rehabil 2014; 93: 938-47.

26. Franki I, Van den Broeck C, De Cat J, et al. A randomized, single-blind cross-over design evaluating the effectiveness of an individually defined, targeted physical therapy approach in treatment of children with cerebral palsy. Clin Rehabil 2014; 28: 1039-52.

27. Abd El-Kafy EM. The clinical impact of orthotic correction of lower limb rotational deformities in children with cerebral palsy: a randomized controlled trial. Clin Rehabil 2014; 28: 1004-14.

28. Grecco LAC, de Almeida Carvalho Duarte N, Mendonça ME, et al. Transcranial direct current stimulation during treadmill training in children with cerebral palsy: a randomized controlled double-blind clinical trial. Res Dev Disabil 2014; 35: 2840-8.

29. Lee B-K, Chon S-C. Effect of whole body vibration training on mobility in children with cerebral palsy: a randomized controlled experimenter-blinded study. Clin Rehabil 2013; 27: 599-607.

30. Dreher T, Götze M, Wolf SI, et al. Distal rectus femoris transfer as part of multilevel surgery in children with spastic diplegia - a randomized clinical trial. Gait Posture 2012; 36: 212-8.

31. Smania N, Bonetti $P$, Gandolfi $M$, et al. Improved gait after repetitive locomotor training in children with cerebral palsy. Am J Phys Med Rehabil Assoc Acad Physiatr 2011; 90: 137-49.

32. van der Houwen LEE, Scholtes VA, Becher JG, Harlaar J. Botulinum toxin A injections do not improve surface EMG patterns during gait in children with cerebral palsy - a randomized controlled study. Gait Posture 2011; 33: 147-51.

33. Johnston TE, Watson KE, Ross SA, et al. Effects of a supported speed treadmill training exercise program on impairment and function for children with cerebral palsy. Dev Med Child Neurol 2011; 53: 742-50.

34. McGibbon NH, Benda W, Duncan BR, Silkwood-Sherer D. Immediate and long-term effects of hippotherapy on symmetry of adductor muscle activity and functional ability in children with spastic cerebral palsy. Arch Phys Med Rehabil 2009; 90: 966-74.

35. Smith PA, Hassani S, Graf A, et al. Brace evaluation in children with diplegic cerebral palsy with a jump gait pattern. J Bone Joint Surg Am 2009; 91: 356-65. 
36. Al-Abdulwahab SS, Al-Khatrawi WM. Neuromuscular electrical stimulation of the gluteus medius improves the gait of children with cerebral palsy. NeuroRehabilitation 2009; 24: 209-17.

37. Seniorou M, Thompson N, Harrington M, Theologis T. Recovery of muscle strength following multilevel orthopaedic surgery in diplegic cerebral palsy. Gait Posture 2007; 26: 475-81.

38. McNee AE, Will E, Lin J-P, et al. The effect of serial casting on gait in children with cerebral palsy: preliminary results from a crossover trial. Gait Posture 2007; 25: 463-8.

39. Engsberg JR, Ross SA, Collins DR. Increasing ankle strength to improve gait and function in children with cerebral palsy: a pilot study. Pediatr Phys Ther Off Publ Sect Pediatr Am Phys Ther Assoc 2006; 18: 266-75.

40. Patikas D, Wolf SI, Mund K, Armbrust P, Schuster W, Döderlein L. Effects of a postoperative strength-training program on the walking ability of children with cerebral palsy: a randomized controlled trial. Arch Phys Med Rehabil 2006; 87: 619-26.

41. Kay RM, Rethlefsen SA, Fern-Buneo A, Wren TAL, Skaggs DL. Botulinum toxin as an adjunct to serial casting treatment in children with cerebral palsy. J Bone Joint Surg Am 2004; 86-A: 2377-84.

42. Bottos M, Benedetti MG, Salucci P, Gasparroni V, Giannini S. Botulinum toxin with and without casting in ambulant children with spastic diplegia: a clinical and functional assessment. Dev Med Child Neurol 2003; 45: 758-62.

43. Desloovere K, Molenaers G, Jonkers I, et al. A randomized study of combined botulinum toxin type $A$ and casting in the ambulant child with cerebral palsy using objective outcome measures. Eur J Neurol Off J Eur Fed Neurol Soc 2001; 8 Suppl 5: 75-87.

44. Graubert C, Song KM, McLaughlin JF, Bjornson KF. Changes in gait at 1 year post-selective dorsal rhizotomy: results of a prospective randomized study. J Pediatr Orthop 2000; 20: 496-500.

45. Ferrari A, Brunner R, Faccioli S, Reverberi S, Benedetti MG. Gait analysis contribution to problems identification and surgical planning in CP patients: an agreement study. Eur J Phys Rehabil Med 2015; 51: 39-48.

46. Dobson F, Morris ME, Baker R, Graham HK. Gait classification in children with cerebral palsy: a systematic review. Gait Posture 2007; 25: 140-52.

47. Schiariti V, Klassen AF, Cieza A, et al. Comparing contents of outcome measures in cerebral palsy using the International Classification of Functioning (ICF-CY): a systematic review. Eur J Paediatr Neurol EJPN Off J Eur Paediatr Neurol Soc 2014; 18: 1-12.

48. Pataky TC, Robinson MA, Vanrenterghem J. Vector field statistical analysis of kinematic and force trajectories. J Biomech 2013; 46: 2394-401.

49. Knudson D, Lindsey C. Type I and Type II Errors in Correlation Analyses of Various Sample Sizes. Med Sci SPORTS Exerc 2013; 45: 328-328.

50. Chau T. A review of analytical techniques for gait data. Part 1: fuzzy, statistical and fractal methods. Gait Posture 2001; 13: 49-66.

51. Rathinam C, Bateman A, Peirson J, Skinner J. Observational gait assessment tools in paediatrics--a systematic review. Gait Posture 2014; 40: 279-85.

52. Harvey A, Gorter JW. Video gait analysis for ambulatory children with cerebral palsy: Why, when, where and how! Gait Posture 2011; 33: 501-3.

53. Baker R, McGinley JL, Schwartz MH, et al. The gait profile score and movement analysis profile. Gait Posture 2009; 30: 265-9. 
54. Chambers HG, Sutherland DH. A practical guide to gait analysis. J Am Acad Orthop Surg. 2002; 10: 222-31.

55. Robertson DG, Winter DA. Mechanical energy generation, absorption and transfer amongst segments during walking. J Biomech 1980; 13: 845-54.

56. Kotiadis D, Hermens HJ, Veltink PH. Inertial Gait Phase Detection for control of a drop foot stimulator Inertial sensing for gait phase detection. Med Eng Phys 2010; 32: 287-97.

57. Schutte LM, Narayanan U, Stout JL, Selber P, Gage JR, Schwartz MH. An index for quantifying deviations from normal gait. Gait Posture 2000; 11: 25-31. 
FIGURES

Figure 1. Study flow diagram. WoS $=$ Web of Science, $I C=$ Inclusion Criteria.

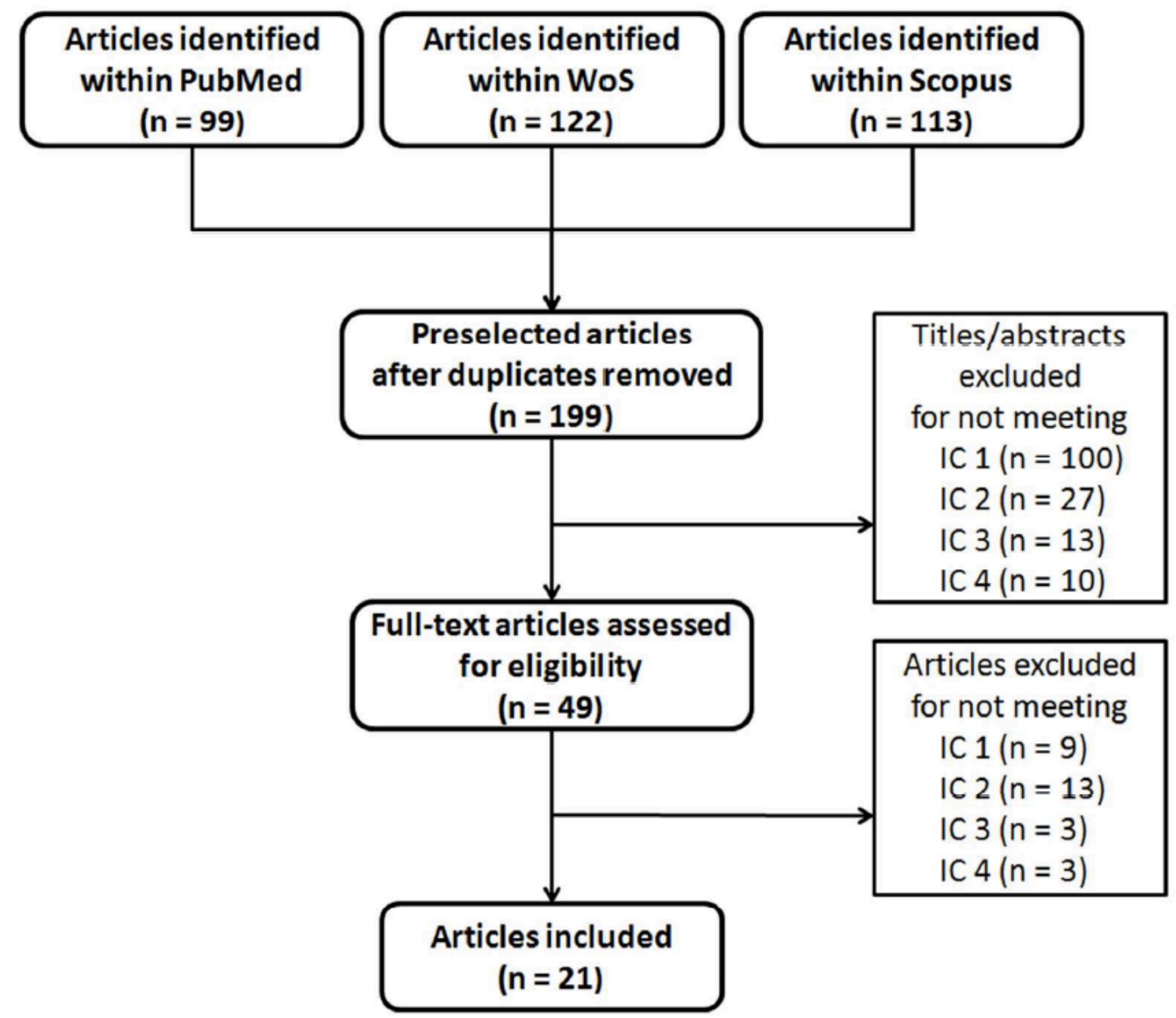




\section{TABLES}

Table 1. Participants' characteristics and selection risk of bias

Table 1

Participants' characteristics and selection risk of bias \begin{tabular}{lcc}
\hline Study & $\begin{array}{c}\text { Sample size } \\
(\mathrm{n})\end{array}$ & $\begin{array}{c}\text { Mean age } \\
(\mathrm{yr} / \mathrm{mo})\end{array}$ \\
& &
\end{tabular} Sex Diagnosis GMFCS Participants's selection risk of bias

Neto et al. (2014) Abd El-Kafy et al. (2014)

Franki et al. (2014) Grecco et al. (2014) Lee et al. (2013) Dreher $(2013)$ Smania et al. (2011)

Van der Houwentat (2011)

Johnston et al. $(2011)^{33}$

McGibbon et al. (2009)

Smith et al. (2009) $)^{35} \wedge$

Al-Abdulwahab et al. (2009)

Seniorou et at (2007) 37

McNee et al. (2007) 3$)^{38}$

Engsberg et al. (2006)

Patikas et al. (2006) ${ }^{40}$

Kay et al. $(2004)^{41}$

Bottos et al. (2003)

Desloovere et al. (2001)

Graubert et al. $(2000)^{44}$

$\begin{array}{lll}5 & 5 & 8\end{array}$

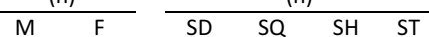

GMFCS = G

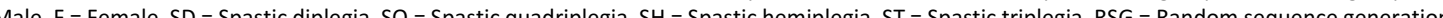

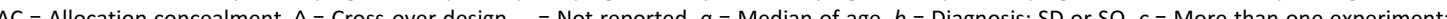
group, $d=$ Independent walking, $e=$ Diagnosis: Mixed, $f=$ Independent or aided walking, $g=$ Nonambulator, assisted ambulatory or independent

ambulatory $+=$ Low risk of bias, ? = unclear risk of bias, $-=$ High risk of bias. 
Table 2. Characteristics of the studies included in the review.

Table 2

Characteristics of the studies included in the review

\begin{tabular}{|c|c|c|c|c|c|c|}
\hline \multirow[t]{2}{*}{ Study } & \multicolumn{2}{|c|}{ Intervention } & \multirow[t]{2}{*}{ Assessment timing } & \multicolumn{2}{|r|}{ IGA } & \multirow{2}{*}{$\begin{array}{c}\text { Gait parameters' } \\
\text { selection risk of bias }\end{array}$} \\
\hline & $\mathrm{E}$ & c & & Measurement tool & Data type & \\
\hline Neto et al. (2014) ${ }^{24}$ & $\mathrm{PI}$ & Placebo & Barefoot/Shoes/+Insoles & 3DGA & Spatiotemporal & \\
\hline Abd El-Kafy et al. (2014) & $\mathrm{BT}+\mathrm{CT}$ & CT & Pre/Post & 3DGA+Video & Spatiotemporal & - \\
\hline Franki et al. $(2014)^{26 \wedge}$ & ITP & GTP & Pre/Post & $3 D G A+F P+S E M G$ & $\begin{array}{l}\text { Spatiotemporal, kinematics, } \\
\text { summary indexes }\end{array}$ & + \\
\hline Abd El-Kafy $(2014)^{27}$ & $\mathrm{CT}+\mathrm{SS} /+\mathrm{SAFO}{ }^{c}$ & CT & Pre/Post & 3DGA+Video & Spatiotemporal, kinematics & + \\
\hline Grecco et al. $(2014)^{28}$ & $\pi T+\mathrm{tDCS}$ & TT+Placebo & Pre/Post/1month & 3DGA+Video & $\begin{array}{l}\text { Spatiotemporal, kinematics, } \\
\text { summary indexes }\end{array}$ & + \\
\hline Lee et al. $(2013)^{29}$ & WBVT+CT & CT & Pre/Post & 3DGA & Spatiotemporal, kinematics & - \\
\hline Dreher et al. $(2012)^{30}$ & SEMLS+CT & SEMLS+DRFT+CT & Pre/1year & 3DGA+FP & $\begin{array}{l}\text { Spatiotemporal, kinematics, } \\
\text { summary indexes }\end{array}$ & - \\
\hline Smania et al. $(2011)^{31}$ & GT & CT & Pre/Post/1month & 3DGA & Spatiotemporal, kinematics & - \\
\hline Van der Houwen et al. $(2011)^{32}$ & $B T X-A+C R$ & CT & Pre/6weeks & Video+sEMG & SEMG & + \\
\hline Johnston et al. (2011) & PBWSTT & CT & Pre/Post/1month & 3DGA & Spatiotemporal & - \\
\hline McGibbon et al. (2009) & HT & BS & Pre/Post & Video+sEMG & SEMG & + \\
\hline Smith et al. $(2009)^{35} \wedge$ & DAFO & HAFO & Barefoot/DAFO/HAFO & 3DGA+Video+FP & $\begin{array}{l}\text { Spatiotemporal, kinematics, } \\
\text { kinetics }\end{array}$ & - \\
\hline Al-Abdulwahab et al. $(2009)^{36}$ & NMES & & Pre/NMES/Post & 3DGA & Spatiotemporal & - \\
\hline Seniorou et al. $(2007)^{37}$ & SEMLS+CT+RS & SEMLS+CT+AE & Pre/Post/1year & 3DGA & Spatiotemporal, kinematics & - \\
\hline McNee et al. $(2007)^{38} \wedge$ & CAST & & Pre/Post & $3 D G A+F P$ & $\begin{array}{l}\text { Spatiotemporal, kinematics, } \\
\text { summary indexes }\end{array}$ & - \\
\hline Engsberg et al. $(2006)^{39}$ & D-ST/P-ST/DP-ST ${ }^{c}$ & & Pre/Post & 3DGA+Video+FP & $\begin{array}{c}\text { Spatiotemporal, kinematics, } \\
\text { kinetics }\end{array}$ & - \\
\hline Patikas et al. $(2006)^{40}$ & SEMLS+CT+ST & $\mathrm{SEMLS}+\mathrm{CT}$ & Pre/1year/2years & $3 D G A+F P$ & $\begin{array}{l}\text { Spatiotemporal, kinematics, } \\
\text { kinetics, summary indexes }\end{array}$ & - \\
\hline Kay et al. $(2004)^{41}$ & BTX-A+CAST & CAST & Pre/3months/1year & 3DGA & Kinematics & - \\
\hline Bottos et al. $(2003)^{42}$ & BTX-A+CAST+CT & $B T X-A+C T$ & Pre/1month/4months & $3 D G A+F P+S E M G$ & $\begin{array}{l}\text { Spatiotemporal, kinematics, } \\
\text { kinetics, sEMG }\end{array}$ & - \\
\hline Desloovere et al. $(2001)^{43}$ & CAST post BTX-A+CT & CAST pre BTX-A+CT & Pre/2months & $3 D G A+V i d e o+F P+s E M G$ & $\begin{array}{l}\text { Spatiotemporal, kinematics, } \\
\text { kinetics, sEMG }\end{array}$ & - \\
\hline
\end{tabular}

Experimental group, $\mathrm{C}=$ Control group, $\mathrm{IGA}=$ Instrumented gait analysis. ${ }^{\wedge}=$ Cross-over design, $c=$ More than one experimental group. $\mathrm{PI}=$ Postural insole, $\mathrm{BT}=$ Balance training, $\mathrm{CT}=$ Conventional therapy, ITP = INdividualized therapy program, $G$ TP = General therapy program, $S S=$ Strapping system, SAFO = Static ankle foot orthosis, $T=$ Treatmill training, tDCS $=$ Transcrania $C R=$ Comprehensive rohabilitation, PBWSTT = Partial body-weight-supported treadmill training HT = Hippotherapy, BS = Barrel-sitting DAFO = Dynamic ankle foot orthosis, HAFO = Hinged ankle foot orthens, NMES = Neuromuscul e ectrical stimultion RS = Resistance stren then strength trainin, DP-ST = Dorsi-and plantarflexion strength training ST = Strength trainin SDR = Selective dorsal rhizotomy. Pre = Pre-intervention assessment. Post = Post-intervention

assessment. $3 \mathrm{DGA}=$ Three dimensional gait analysis, $\mathrm{FP}=$ Force plate, $\mathrm{SEMG}=$ Surface electromyography. $+=$ Low risk of bias, $?=$ unclear risk of bias, $-=$ High risk of bias. 
Table 3

Gait parameters definitions

\begin{tabular}{|c|c|c|}
\hline Type & Nomenclature & Definition \\
\hline \multicolumn{3}{|c|}{ SPATIOTEMPORAL } \\
\hline & Gait speed & Mean velocity of progression in longitudinal direction. In meters/second. ${ }^{28}$ \\
\hline & Cadence & Number of steps in a time unit. In steps/minute. ${ }^{28}$ \\
\hline & Stride length & Longitudinal distance between successive points of heel contact of the same foot. In meters. ${ }^{28}$ \\
\hline & Step width & Distance between the rear end of the right and left heel centerlines along the mediolateral axis. In meters. ${ }^{28}$ \\
\hline & Time of toe off & Instant in the gait cycle in which toe off occurs. It also refers to the duration of stance phase. In percentage of gait cycle. \\
\hline & Single support & Percentage of the gait cycle in which one foot is in contact with the floor. ${ }^{14}$ It includes MSt and TSt. \\
\hline & Double support & Percentage of the gait cycle in which both feet are in contact with the floor. There are two double support periods during a gait cycle (LR and PSw). ${ }^{14}$ \\
\hline \multicolumn{3}{|c|}{$\begin{array}{l}\text { KINEMATIC AND KINETIC } \\
\text { Value }\end{array}$} \\
\hline & MAX & Maximum value. ${ }^{20}$ In degrees (angle), $\mathrm{N} \cdot \mathrm{m}$ (moment) and $\mathrm{W}$ (power). \\
\hline & MIN & Minimum value. ${ }^{20}$ In degrees (angle), $\mathrm{N} \cdot \mathrm{m}$ (moment) and $\mathrm{W}$ (power). \\
\hline & MAPO & Temporal position of the maximum value. ${ }^{20}$ In percentage of gait cycle. \\
\hline & MIPO & Temporal position of the minimum value. ${ }^{20}$ In percentage of gait cycle. \\
\hline & ROM & Range of motion (MAX-MIN). ${ }^{20}$ In degrees (angle), N.m (moment) and W (power). \\
\hline & MEAN & $\begin{array}{l}\text { Mean value }{ }^{20} \text {, in degrees (angle), } \mathrm{N} \cdot \mathrm{m} \text { (moment) and } \mathrm{W} \text { (power), calculated as: } M E A N_{i}=\frac{1}{T} \sum_{t=1}^{T} x_{i, t} \text { where } \mathrm{x}_{\mathrm{i}, \mathrm{t}} \text { is the value of a gait variable } i \text { at a specific } \\
\text { instant } t \text { in the gait cycle, and } \mathrm{T} \text { is the number of instants into which the gait cycle was divided. }\end{array}$ \\
\hline & GVS & $\begin{array}{l}\text { The Gait Variable Score is the root mean square (RMS) difference between a normalized temporal kinematic variable (joint or segment angle) and the } \\
\text { average kinematic variable from a reference group, calculated point-by-point across the gait cycle }{ }^{26,28,53}: G V S_{i}=\sqrt{\frac{1}{T} \sum_{t=1}^{T}\left(x_{i, t}-\bar{x}_{i, t}^{r e f}\right)^{2}} \text { where } x_{i, t} \text { is the } \\
\text { value of a gait variable } i \text { at a specific instant } t \text { in the gait cycle, } \bar{x}_{i, t}^{r e f} \text { is the mean value of that variable at the same instant for the reference population, } \\
\text { and T is the number of instants into which the gait cycle was divided. In degrees. }\end{array}$ \\
\hline
\end{tabular}

\section{Time-series}

Foot kinematics

FootPro FootInExRot

Ankle kinematics

DorsPlantFlex Ankle dorsi-plantar flexion angle in the sagittal plane.

Knee kinematics

KneeFlexExt Knee flexion-extension angle in the sagittal plane.

KneeFlexExtVe

Knee flexion-extension velocity in the sagittal plane. It can be calculated as the temporal gradient (slope) of the KneeFlexExt angle: $v_{i, t}=\frac{1}{2}\left(x_{i, t+1}-x_{i, t-1}\right)$ where $\mathrm{x}_{\mathrm{i}, \mathrm{t}}$ is the value of a gait variable $i$ at a specific instant $t$ in the gait cycle. ${ }^{20}$

Hip kinematics

HipFlexExt Hip flexion-extension angle in the sagittal plane.

HipAddAbd Hip adduction-abduction angle in the frontal plane.

HiplnExRot Hip internal-external rotation angle in the transverse plane.

Pelvis kinematics

PelvicTilt Pelvic tilt orientation in the sagittal plane.

PelvicObl Pelvic obliquity orientation in the frontal plane.

PelvicRot Pelvic rotation orientation in the transverse plane.

Ankle kinetics

PlantDorsFlexMo Internal ankle moment in the sagittal plane. It indicates muscle activity of plantar-flexors (positive values) and dorsi-flexors (negative values). ${ }^{54}$

AnkleGenAbsPo Ankle power in the sagittal plane. Generation power indicates concentric contraction and absorption power indicates eccentric contraction. ${ }^{55}$

Knee kinetics

KneeGenAbsPo Knee power in the sagittal plane. Generation power indicates concentric contraction and absorption power indicates eccentric contraction. ${ }^{55}$

Hip Kinetics

Gait phase

HipGenAbsPo Hip power in the sagittal plane. Generation power indicates concentric contraction and absorption power indicates eccentric contraction. ${ }^{55}$

Events

IC Initial contact is the instant in which the initial foot strike occurs ( $0 \%$ of gait cycle). ${ }^{54}$

ForeAftShear Instant in which reversal of fore to aft shear occurs. ${ }^{54}$

TOff Instant in which toe off occurs ( $\approx 62 \%$ of gait cycle). ${ }^{54}$

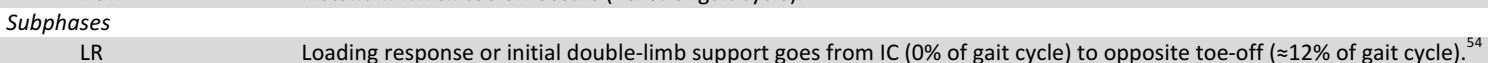

MSt $\quad$ Midstance refers to initial single-limb stance and goes from opposite toe-off ( $\approx 12 \%$ of gait cycle) to ForeAftShear $r^{54}$ (or heel off if it occurs).

TSt Terminal stance refers to terminal single-limb stance and goes from ForeAftShear (or heel off if it occurs) to opposite foot strike ( $\approx 50 \%$ of gait cycle). ${ }^{54}$

PSw Preswing or second double-limb support goes from opposite foot strike ( $\approx 50 \%$ of gait cycle) to TOff. ${ }^{54}$

ISW Initial swing goes from TOff to foot clearance $\left(\approx 75 \%\right.$ of gait cycle) ${ }^{54}$

MSw Midswing goes from foot clearance $\left(\approx 75 \%\right.$ of gait cycle) to tibia vertical ( $\approx 85 \%$ of gait cycle). ${ }^{54}$

TSw Terminal swing goes from tibia vertical $\left(\approx 85 \%\right.$ of gait cycle) to second foot strike $\left(100 \%\right.$ of gait cycle). ${ }^{54}$

Phases

St Stance is the phase in which the foot is in contact with the floor. It is from IC to TOff. It lasts for about $62 \%$ of gait cycle. ${ }^{54}$

POff Push off goes from ForeAftShear (or heel off if it occurs) to TOff. ${ }^{56}$ It includes TSt and PSw and it is part of the Stance phase.

Sw Swing is the phase in which the foot is not in contact with the floor. It is from TOff to second foot strike. It lasts for about $38 \%$ of gait cycle. ${ }^{54}$

Gait cycle

Stri Stride is the movement from one foot strike (initial) to the successive foot strike (second) on the same side. ${ }^{54}$

SUMMARY INDEXES

GGI

The Gillette Gait Index, also called the Normalcy Index, uses multivariate statistical methods to quantify the deviation of a subject's gait from an unimpaired control group. It is calculated from three spatiotemporal parameters (timing of toe off, gait speed normalized by leg length and cadence) and 13 kinematic parameters (MEAN_PelvicTilt_Stri, ROM_PelvicTilt_Stri, MEAN_PelvicRot_Stri, MIN_HipFlexExt_Stri, ROM_HipFlexExt_Stri, MIN_HipAddAbd_Sw, MEAN_HipInExRot_St, KneeFlexExt_IC, MAPO_KneeFlexExt_Stri, ROM_KneeFlexExt_Stri, MAX_DorsPlantFlex_St, MAX_DorsPlantFlex_Sw and MEAN_FootPro_Stri). ${ }^{18}$ Schutte et al ${ }^{57}$ described its calculation.

nine GVS scores for that side ${ }^{18,28}: G P S=\sqrt{\frac{1}{N} \sum_{i=1}^{N} G V S_{i}^{2}}$. In degrees. 
Table 4. Instrumented gait analysis: Spatiotemporal, kinetic, sEMG and segment angles parameters and summary indexes.

\section{Table 4}

\begin{tabular}{|c|c|c|c|c|c|c|c|c|c|c|c|c|c|c|c|c|c|c|c|c|c|c|c|c|c|c|c|c|c|c|c|c|c|c|c|}
\hline \multirow[t]{4}{*}{ Study } & \multirow{3}{*}{\multicolumn{6}{|c|}{ Spatiotemporal }} & \multicolumn{8}{|c|}{ 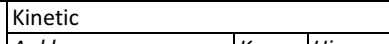 } & \multirow{3}{*}{\multicolumn{6}{|c|}{ sEMG }} & \multicolumn{13}{|c|}{ Segment kinematic } & & \\
\hline & & & & & & & \multirow{2}{*}{\multicolumn{5}{|c|}{$\begin{array}{r}\text { Ankle } \\
\text { Mo }\end{array}$}} & \multirow{3}{*}{ 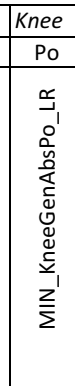 } & \multirow{2}{*}{\multicolumn{2}{|c|}{\begin{tabular}{|l} 
Hip \\
Po \\
\end{tabular}}} & & & & & & & \multirow{2}{*}{\multicolumn{4}{|c|}{\begin{tabular}{|l|}
\multicolumn{2}{|l|}{ Foot } \\
FP \\
\end{tabular}}} & \multirow{2}{*}{\multicolumn{9}{|c|}{\begin{tabular}{|r|} 
Pelvis \\
SP \\
\end{tabular}}} & & \\
\hline & & & & & & & & & & & 0 & & & & & & & & & & & & & & & & & & & & & & & & \\
\hline & 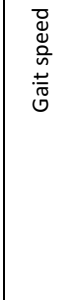 & 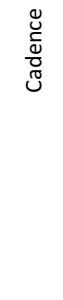 & 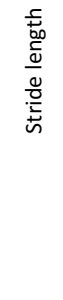 & 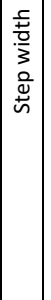 & 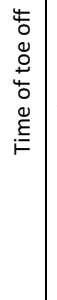 & 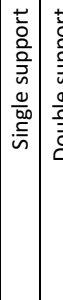 & 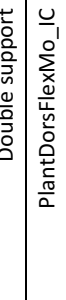 & 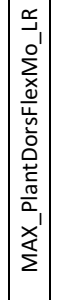 & 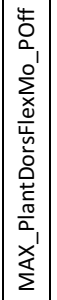 & 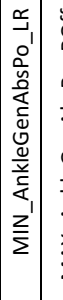 & 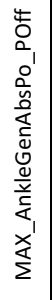 & & 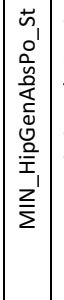 & 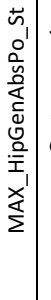 & 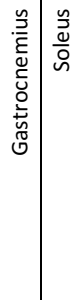 & 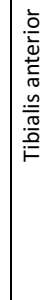 & 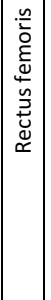 & 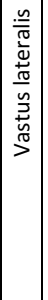 & 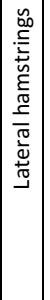 & 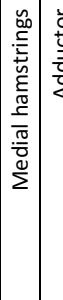 & 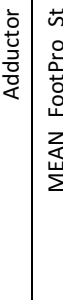 & 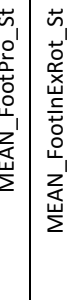 & 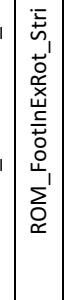 & 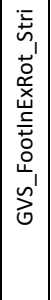 & 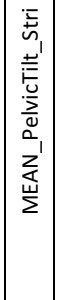 & 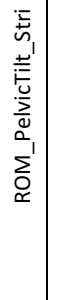 & 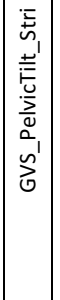 & 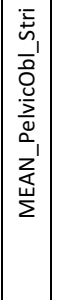 & 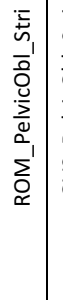 & 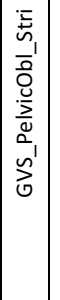 & 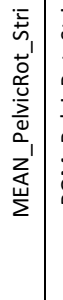 & 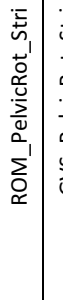 & 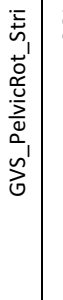 & $\overline{\mathrm{J}}$ & 号 \\
\hline Neto et al. (2014) & $E^{*}$ & $\mathrm{E}^{*}$ & $\circ$ & & 0 & & o & & & & & & & & & & & & & & & & & & & & & & & & & & & & \\
\hline Abd El-Kafy et al. (2014) & $\mathrm{EC}^{*}$ & EC* & $\mathrm{EC}^{*}$ & & EC* & & & & & & & & & & & & & & & & & & & & & & & & & & & & & & \\
\hline Franki et al. (2014) & & & $\mathrm{E}^{*}$ & & & & & & & & & & & & & & & & & & & & & 0 & & & 0 & & & C & & & $\mathrm{E}$ & & $\circ$ \\
\hline Abd El-Kafy $(2014)^{27}$ & $\mathrm{EC}^{*}$ & $\mathrm{EC} *$ & $\mathrm{EC} *$ & & & & & & & & & & & & & & & & & & & & & & & & & & & & & & & & \\
\hline Grecco et al. $(2014)^{28}$ & $E^{*}$ & $E^{*}$ & $\circ$ & 0 & $\circ$ & & & & & & & & & & & & & & & & & & & 0 & & & $E^{*}$ & & & 0 & & & $\circ$ & & $E^{*}$ \\
\hline Lee et al. $(2013)^{29}$ & $E$ & $E$ & $E$ & & & & & & & & & & & & 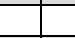 & & 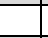 & & & 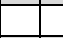 & & & & 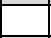 & & & & & & & & & & & \\
\hline Dreher et al. $(2012)^{30}$ & $\circ$ & 으 & $\circ$ & & $\circ$ & & & & & & & & & & & & & & & & & & & & & & & & & & & & & $\mathrm{EC}$ & \\
\hline Smania et al. $(2011)^{31}$ & $E^{*}$ & $\circ$ & $E^{*}$ & & & & & & & & & & & & & & & & & & & & & & & & & & & & & & & & \\
\hline Van der Houwen et al. (2011) & & & & & & & & & & & & & & & $*$ & & $\circ$ & & & 0 & & & & & & & & & & & & & & & \\
\hline \begin{tabular}{|l} 
Johnston et al. (2011) \\
$3^{33}$
\end{tabular} & EC & $\mathrm{c}$ & $E$ & & & & & & & & & & & & & & & & & & & & & & & & & & & & & & & & \\
\hline McGibbon et al. (2009) & & & & & & & & & & & & & & & & & & & & & E* & & & & & & & & & & & & & & \\
\hline Smith et al. $(2009)^{35}$ & EC & $\mathrm{EC}$ & EC & & & & 0 & & EC & & 0 & & & & & & . & & & & & & & & & & & & & & & & & & \\
\hline Al-Abdulwahab et al. (2009) & $E$ & & $E$ & 0 & & & & & & & & & & & & & & & & & & & & & & & & & & & & & & & \\
\hline Seniorou et al. $(2007)^{37}$ & $E$ & & & & & & & & & & & & & & & & & & & & & & & & & & & & & & & $\circ$ & & & \\
\hline McNee et al. $(2007)^{38}$ & $\circ$ & $\circ$ & $\circ$ & & & * & & & - & & & & & & & & & & & & & & & & & & & & & & & & & $\circ$ & \\
\hline Engsberg et al. (2006) & $\circ$ & $\circ$ & $\circ$ & & & & & & 0 & & & & & & & & & & & & & & & & & & & & & & & & & & \\
\hline Patikas et al. $(2006)^{40}$ & $\circ$ & $\circ$ & $\circ$ & & $\circ$ & & & & 0 & & $\circ$ & $\circ$ & 0 & & & & & & & 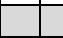 & & & & & & & & & . & & & & & $\circ$ & \\
\hline Bottos et al. $(2003)^{42}$ & * & $\circ$ & * & & $\circ$ & & & $\circ$ & 0 & & & & & & 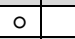 & $\circ$ & & & & & & & & & & & & & & & & & & & \\
\hline Desloovere et al. (2001) & 0 & 0 & 0 & & & & & $\mathrm{EC}$ & EC & \begin{tabular}{|l|l}
$\mathrm{EC}$ & \\
\end{tabular} & 0 & & & \begin{tabular}{l|l}
$C$ & $E$ \\
\end{tabular} & \begin{tabular}{|l|l|} 
EC & EC \\
\end{tabular} & $\mathrm{C}$ & c & 0 & C & EC & & \begin{tabular}{l|l|}
$E$ & $E$ \\
\end{tabular} & & & 0 & 0 & & 0 & 0 & & 0 & $E$ & & & \\
\hline Graubert et al. $(2000)^{44}$ & $\circ$ & $\circ$ & $\circ$ & & & & & & & & & & & & & & & & & & & & $*$ & & & $\circ$ & & & & & & & & & \\
\hline
\end{tabular}


Table 5. Instrumented gait analysis: Joint angles parameters.

Table 5

Instrumented gait analysis: Joint angles parameters

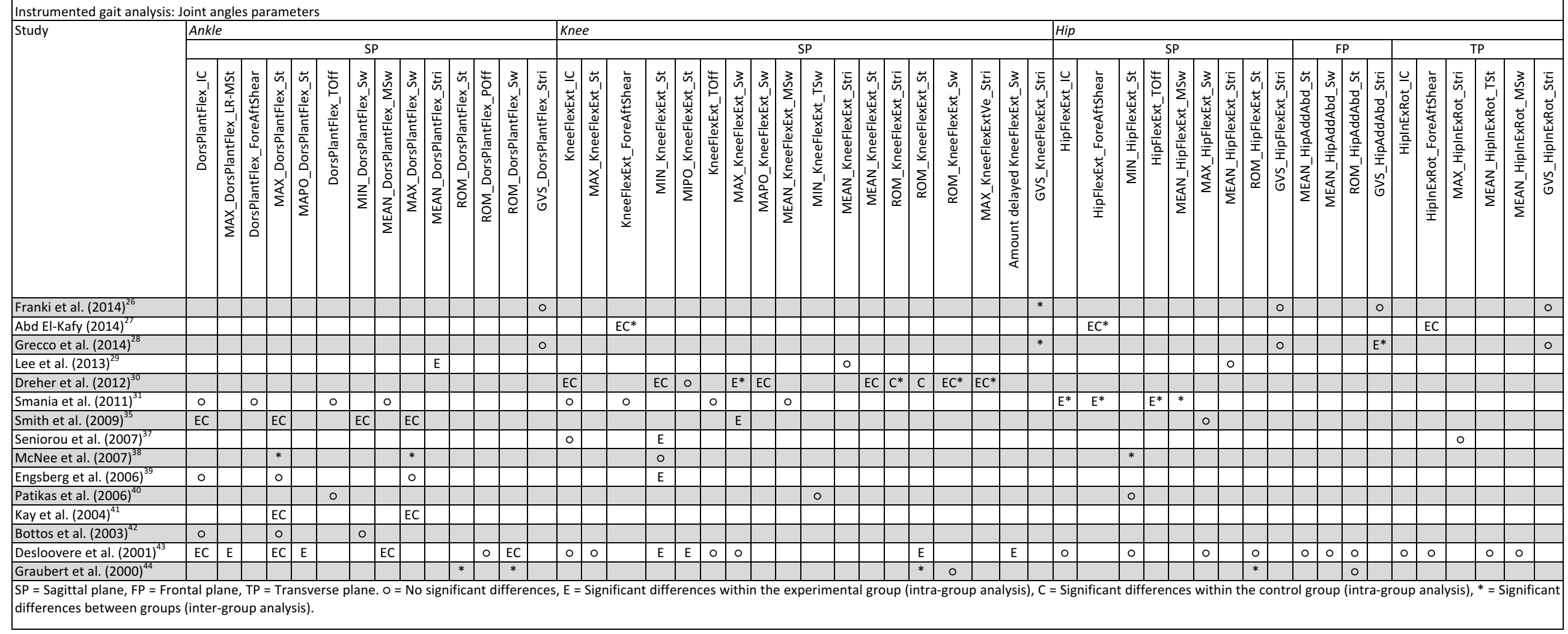


Table 6. Gait parameters responsiveness to different treatments.

Table 6

Gait parameters responsiveness to different treatments.

\begin{tabular}{llcl}
\hline Interventions & & Gait parameters
\end{tabular}

\begin{tabular}{|c|c|c|c|c|c|c|c|c|c|c|c|}
\hline & \multirow{2}{*}{ spatiotemporal } & & \multirow{2}{*}{$\mathrm{SI}$} & & \multirow{2}{*}{ SEIVIG } \\
\hline & & Foot & Ankle & Knee & Hip & Pelvis & & Ankle & Knee & Hip & \\
\hline Surgery ${ }^{30,44}$ & $\circ$ & ROM_FootInExRot_Stri & $\begin{array}{l}\text { ROM_DorsPlantFlex_St, } \\
\text { ROM_DorsPlantFlex_Sw }\end{array}$ & $\begin{array}{l}\text { KneeFlexExt_IC, } \\
\text { MIN_KneeFlexExt_St, } \\
\text { MAX_KneeFlexExt_Sw, } \\
\text { MAPO_KneeFlexExt_Sw, } \\
\text { MEAN_KneeFlexExt_St, } \\
\text { ROM_KneeFlexExt_Stri, } \\
\text { ROM_KneeFlexExt_St, } \\
\text { ROM_KneeFlexExt_Sw, } \\
\text { MAX_KneeFlexExtVe_Stri }\end{array}$ & ROM_HipFlexExt_St & $\circ$ & GGI & $x$ & $x$ & $x$ & $x$ \\
\hline $\begin{array}{l}\text { BTX-A + } \\
\text { Casting }^{32,38,41-43}\end{array}$ & $\begin{array}{l}\text { Gait speed, } \\
\text { Stride length, } \\
\text { Single support }\end{array}$ & $\begin{array}{l}\text { MEAN_FootPro_St, } \\
\text { MEAN_FootInExRot_St }\end{array}$ & $\begin{array}{l}\text { DorsPlantFlex_IC, } \\
\text { MAX_DorsPlantFlex_LR-MSt, } \\
\text { MAX_DorsPlantFlex_St, } \\
\text { MAPO_DorsPlantFlex_St, } \\
\text { MEAN_DorsPlantFlex_MSw, } \\
\text { MAX_DorsPlantFlex_Sw, } \\
\text { ROM_DorsPlantFlex_Sw }\end{array}$ & $\begin{array}{l}\text { MIN_KneeFlexExt_St, } \\
\text { MIPO_KneeFlexExt_St, } \\
\text { ROM_KneeFlexExt_St, }\end{array}$ & MIN_HipFlexExt_St & ROM_PelvicRot_Stri & $\circ$ & $\begin{array}{l}\text { MAX_PlantDorsFlexMo_LR, } \\
\text { MAX_PlantDorsFlexMo_POff, } \\
\text { MIN_AnkleGenAbsPo_LR }\end{array}$ & $\mathrm{x}$ & MAX_HipGenAbsPo_St & $\begin{array}{l}\text { Soleus, } \\
\text { Tibialis anterior, } \\
\text { Gastrocnemius, } \\
\text { Rectus femoris, } \\
\text { Lateral hamstrin } \\
\text { Medial hamstrin }\end{array}$ \\
\hline $\begin{array}{l}\text { Orthopedic } \\
\text { device }{ }^{24,2,3,35}\end{array}$ & $\begin{array}{l}\text { Gait speed, } \\
\text { Cadence, } \\
\text { Stride length }\end{array}$ & $\mathrm{x}$ & $\begin{array}{l}\text { DorsPlantFlex_IC, } \\
\text { MAX_DorsPlantFlex_St, } \\
\text { MIN_DorsPlantFlex_Sw, } \\
\text { MAX_DorsPlantFlex_Sw }\end{array}$ & $\begin{array}{l}\text { KneeFlexExt_ForeAftShear, } \\
\text { MAX_KneeFlexExt_Sw }\end{array}$ & $\begin{array}{l}\text { HipFlexExt_ForeAftShear, } \\
\text { HipInExRot_ForeAftShear }\end{array}$ & $\mathrm{x}$ & $\mathrm{x}$ & MAX_PlantDorsFlexMo_POff & $\mathrm{x}$ & $\mathrm{x}$ & $\mathrm{x}$ \\
\hline $\begin{array}{l}\text { Strength } \\
\text { training } \mathrm{g}^{29,36,37,39,}\end{array}$ & $\begin{array}{l}\text { Gait speed, } \\
\text { Cadence, } \\
\text { Stride length }\end{array}$ & $\mathrm{x}$ & MEAN__DorsPlantFlex_Stri & MIN_KneeFlexExt_St & $\circ$ & $\circ$ & $\circ$ & $\circ$ & $\circ$ & $\circ$ & $\mathrm{x}$ \\
\hline $\begin{array}{l}\text { Balance } \\
\text { training }\end{array}$ & $\begin{array}{l}\text { Gait speed, } \\
\text { Cadence, } \\
\text { Stride length, } \\
\text { Time of toe off }\end{array}$ & $\mathrm{x}$ & $\mathrm{x}$ & $\mathrm{x}$ & $\mathrm{x}$ & $\mathrm{x}$ & $\mathrm{x}$ & $\mathrm{x}$ & $\mathrm{x}$ & $\mathrm{x}$ & $\mathrm{x}$ \\
\hline $\begin{array}{l}\text { Gait } \\
\text { training }^{28,31,33}\end{array}$ & $\begin{array}{l}\text { Gait speed, } \\
\text { Cadence, } \\
\text { Stride length }\end{array}$ & $\circ$ & $\circ$ & GVS_KneeFlexExt_Stri & $\begin{array}{l}\text { HipFlexExt_IC, } \\
\text { HipFlexExt_ForeAftShear, } \\
\text { HipFlexExt_TOff, } \\
\text { MEAN_HipFlexExt_MSw, } \\
\text { GVS_HipAddAbd_Stri }\end{array}$ & GVS_PelvicTilt_Stri & GPS & $x$ & $\mathrm{x}$ & $\mathrm{x}$ & $\mathrm{x}$ \\
\hline ITP 26 & Stride length & 0 & $\circ$ & GVS_KneeFlexExt_Stri & 0 & GVS_PelvicRot_Stri & 0 & $\mathrm{x}$ & $\mathrm{x}$ & $\mathrm{x}$ & $\mathrm{x}$ \\
\hline Hippotherapy ${ }^{34}$ & $x$ & $\mathrm{x}$ & $\mathrm{x}$ & $x$ & $\mathrm{x}$ & $x$ & $\mathrm{x}$ & $\mathrm{x}$ & $\mathrm{x}$ & $\mathrm{x}$ & Adductor \\
\hline
\end{tabular}

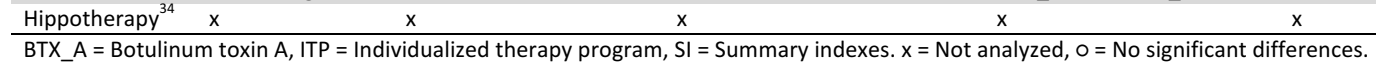

